\title{
EXTREMAL QUASICONFORMAL MAPPINGS OF A CONE
}

\author{
OSSI TAARI
}

In the paper [2] we proved the following extremal property for cylinder: Let $G$ be a domain in $R^{n-1}$ with $m_{n-1}(G)<\infty$ and $f$ a quasiconformal mapping of the cylinder $Z=G \times R^{1} \subset R^{n}$ onto itself which satisfies the boundary condition

$$
f\left(x_{1}, \ldots, x_{n}\right)=\left(x_{1}, \ldots, x_{n-1}, K x_{n}\right),
$$

where $K \geqq 1$ is a constant. Then $K_{O}(f) \geqq K^{n-1}$ and $K_{I}(f) \geqq K$, where $K_{O}(f)$ and $K_{I}(f)$ are the outer and inner dilatations of the mapping $f$. In the extremal case $K_{O}(f)=K^{n-1}$ the lines parallel to $x_{n}$-axis go to similar lines, and the image of the section

$$
G(t)=\left\{x+t e_{n} \mid x \in G\right\}
$$

of $Z$ is for every $t \in R^{1}$ the section $G(K t)$. However, the mapping need not then be affine. On the other hand, if $K_{I}(f)=K$, then $f$ is the affine mapping (1).

Now we consider a similar problem for cones. Let $G$ be a domain in $S^{n-1}, \quad G \neq S^{n-1}$, and let $C$ be the cone $\left\{x \in R^{n}|x /| x \mid \in G\right\}$. We consider a homeomorphism $f: \bar{C} \rightarrow \bar{C}$ whose restriction to $C$ is quasiconformal and which satisfies on the boundary $\partial C$ the condition

$$
f(x)=|x|^{K-1} x,
$$

where $K \geqq 1$ is a constant.

By Rickman [1], Theorem 1, or Väisälä [4], Theorem 2, $f$ can be extended to a quasiconformal mapping $\hat{f}: R^{n} \rightarrow R^{n}$ so that

$$
\hat{f}(x)=\left\{\begin{array}{l}
f(x), \text { when } x \in C \\
|x|^{K-1} x, \text { when } x \in R^{n} \backslash C .
\end{array}\right.
$$

The following distortion theorem is valid for $f$. 
Th e o r e m 1. Suppose that $f: C \rightarrow C$ is a quasiconformal mapping which satisfies the boundary condition (2). Then there exist positive constants $\lambda$ and $M>1$ such that

$$
|x|^{K} / \lambda \leqq|\hat{f}(x)| \leqq \lambda|x|^{K}
$$

holds for $|x| \geqq M$ or $|x| \leqq 1 / M$. Here $\lambda$ depends only on $K$ and $K(f)$.

Proof. Denote $\lambda=2 H(0, \hat{f})$, where $H(0, f)$ is the linear dilatation of $\hat{f}$ in the origin. The assertion for small values of $|x|$ follows then immediately.

For big values the result follows from the preceding one by inversion $\varphi(x)=x /|x|^{2}$. Now $\varphi^{-1} \circ f \circ \varphi$ is a quasiconformal mapping of $R^{n}$ with the same dilatations and values on $\partial C$ as $f$ itself.

In the following theorem we give the natural lower bounds for the dilatations.

The o r e m 2. If $f$ satisfies the boundary condition (2), then

$$
K_{O}(f) \geqq K^{n-1}, K_{I}(f) \geqq K .
$$

Proof. Choose $0<r_{1}<1 / M<M<r_{2}$ such that $\lambda r_{1}^{K}<r_{2}^{K} / \lambda$. Let $\Gamma$ be the curve family which joins the sets $G\left(r_{1}\right)=r_{1} G=$ $\left\{x|x| r_{1} \in G\right\}$ and $G\left(r_{2}\right)=r_{2} G$ in $C$. Then by Sections 7.7 and 6.4 of $[3]$

$$
M(\Gamma)=m_{n-1}(G) /\left(\log \left(r_{2} / r_{1}\right)\right)^{n-1}
$$

and

$$
M(f \Gamma) \leqq m_{n-1}(G) /\left(\log \left(\lambda^{-1} r_{2}^{K} / \lambda r_{1}^{K}\right)\right)^{n-1}
$$

Letting $r_{2} \rightarrow \infty$ we obtain by $M(\Gamma) \leqq K_{0}(f) M(f \Gamma)$

$$
K_{o}(f) \geqq K^{n-1} \text {. }
$$

Since $K_{O}(f) \leqq K_{I}(f)^{n-1}$, we have

$$
K_{I}(f) \geqq K .
$$

Theorem 3. If $K_{O}(f)=K^{n-1}$, then $f$ maps each ray $r(y)=$ $\{t y \mid t>0\}, y \in G$, onto a similar ray, and the image of

$$
G(t)=t G
$$

is for every $t>0$ the set $G\left(t^{K}\right)$. Further, the volume derivative $\sigma\left(x, h_{t}\right)$ of the homeomorphism $h_{t}=f \mid G(t)$ is equal to $t^{(n-1)(K-1)}$ for almost every $x \in G(t)$.

Before the proof of Theorem 3 we introduce some preliminary lemmas.

$\mathrm{L}$ e m m a 1 . If $K_{o}(f)=K^{n-1}$, then 


$$
0 \leqq \int_{C}\left(L_{f}(x)^{n}-\left|\partial_{x}\right| f(x)||^{n}\right)|f(x)|^{-n} \leqq 2 \log \lambda(1+n) K^{n-1} m_{n-1}(G)
$$

where $\partial_{x}|f(x)|$ is the directional derivative of $|f|$ at $x$ in the direction of $x$.

Proof. Let $j \in N, j>M$ and $j^{K}>\lambda$; then Theorem 1 is valid for $|x|=j$, and $j^{K} / \lambda>\lambda / j^{K}$. On almost every ray $r(y)=\{t y \mid t>0\}$, $y \in G, f$ and thus also $|f|$ are locally absolutely continuous. Moreover, $f$ is differentiable at almost all points of $r(y)$. Theorem 1 implies

$$
\begin{gathered}
\log \left(j^{K} / \lambda\right)-\log \left(\lambda / j^{K}\right) \leqq \log |f(j y)|-\log |f(y \mid j)| \\
=\int_{1 / j}^{j}\left(\partial_{y}|f(t y)| /|f(t y)|\right) d t .
\end{gathered}
$$

By Hölder's inequality we obtain

$$
\begin{aligned}
(2 K \log j- & 2 \log \lambda)^{n} \leqq\left.(2 \log j)^{n-1} \int_{1 / j}^{j}\left|\partial_{y}\right| f(t y)\left|{ }^{n}\right| f(t y)\right|^{-n} t^{n-1} d t \\
& \leqq(2 \log j)^{n-1} \int_{1 / j}^{j} L_{|f|}(t y)^{n}|f(t y)|^{-n} t^{n-1} d t \\
& \leqq(2 \log j)^{n-1} \int_{1 / j}^{j} L_{f}(t y)^{n}|f(t y)|^{-n} t^{n-1} d t
\end{aligned}
$$

the inequality $L_{|f|}(t y) \leqq L_{f}(t y)$ follows from the triangle inequality. Since $L_{f}(x)^{n} \leqq K_{O}(f) J(x, f)$ at almost every point of

$$
C_{j}=\{x \in C|1 / j \leqq| x \mid \leqq j\},
$$

if follows by integrating over $G$

$$
\begin{aligned}
& (2 K \log j-2 \log \lambda)^{n} m_{n-1}(G) \\
\leqq & (2 \log j)^{n-1} \int_{C_{j}}\left|\partial_{x}\right| f(x)||^{n}|f(x)|^{-n} d m(x) \\
\leqq & (2 \log j)^{n-1} \int_{C_{j}} L_{f}(x)^{n}|f(x)|^{-n} d m(x) \\
\leqq & (2 \log j)^{n-1} K^{n-1} \int_{C_{j}} J(x, f)|f(x)|^{-n} d m(x) \\
= & (2 \log j)^{n-1} K^{n-1} \int_{f C_{j}}|z|^{-n} d m(z) \\
\leqq & (2 K \log j)^{n-1} \int_{G} d m_{n-1} \int_{\lambda^{-1} j_{j}^{K}} t^{-1} d t \\
= & (2 K \log j)^{n-1} m_{n-1}(G)(2 K \log j+2 \log \lambda) .
\end{aligned}
$$

This implies 


$$
\begin{aligned}
0 & \leqq \int_{C_{j}}\left(L_{f}(x)^{n}-\left|\partial_{x}\right| f(x)||^{n}\right)|f(x)|^{-n} d m(x) \\
& \leqq m_{n-1}(G)\left(2(n+1) \log \lambda K^{n-1}+\varepsilon_{j}\right),
\end{aligned}
$$

where $\lim _{j \rightarrow \infty} \varepsilon_{j}=0$. Letting $j \rightarrow \infty$ yields the lemma.

$\mathrm{L}$ e m m a 2. If $K_{0}(f)=K^{n-1}$, then the function

$$
\left(\left(L_{f}-\partial_{x}|f|\right) /|f|\right)^{n}
$$

is integrable in $\mathrm{C}$.

Proof. Let $A$ and $B$ be those subsets of $C$ where $\partial_{x}|f(x)| \geqq 0$, resp. $\partial_{x}|f(x)|<0$. By $0 \leqq \partial_{x}|f(x)| \leqq L_{f}(x)$ the inequality

$$
\left(L_{f}(x)-\partial_{x}|f(x)|\right)^{n} \leqq L_{f}(x)^{n}-\left(\partial_{x}|f(x)|\right)^{n}
$$

holds in $A$ and the integral of $\left(\left(L_{f}-\partial_{x}|f|\right) /|f|\right)^{n}$ over $A$ is finite by Lemma 1.

To obtain the respective result for the set $B$ we prove that

$$
\int_{B} L_{f}(x)^{n}|f(x)|^{-n} d m(x)
$$

is finite. If $j \geqq \mathbf{M}$, then for almost all rays $r_{j}(y)=\{t y \mid 1 / j \leqq t \leqq j\}$, $y \in G$,

$$
\begin{aligned}
& \log \left(j^{K} / \lambda\right)-\log \left(\lambda / j^{K}\right) \leqq \int_{r_{j}(y)}\left(\partial_{y}|f(x)| /|f(x)|\right) d m_{1}(x) \\
\leqq & \int_{r_{j}(y) \cap A}\left(\partial_{y}|f(x)| /|f(x)|\right) d m_{1}(x) \leqq \int_{r_{j}(y) \cap A}\left(L_{f}(x) /|f(x)|\right) d m_{1}(x) .
\end{aligned}
$$

By Hölder's inequality and integration over $G$ we obtain

$$
(2 K \log j-2 \log \lambda)^{n} m_{n-1}(G) \leqq(2 \log j)^{n-1} \int_{C_{j} \cap A} L_{f}(x)^{n}|f(x)|^{-n} d m(x) .
$$

On the other hand,

$$
\int_{C_{j}} L_{f}(x)^{n}|f(x)|^{-n} d m(x) \leqq K^{n-1}(2 K \log j+2 \log \lambda) m_{n-1}(G) .
$$

From these inequalities it follows that

$$
\int_{C_{j} \cap B} L_{f}(x)^{n}|f(x)|^{-n} d m(x) \leqq\left[2(n+1) K^{n-1} \log \lambda+\varepsilon_{j}\right] m_{n-1}(G) .
$$

Letting $j \rightarrow \infty$ yields

$$
\int_{B} L_{f}(x)^{n}|f(x)|^{-n} d m(x) \leqq 2(n+1) K^{n-1} \log \lambda m_{n-1}(G) .
$$

The inequality $\left|\partial_{x}\right| f(x)|| \leqq L_{f}(x)$ implies 


$$
\int_{B}\left(\left(L_{f}(x)-\partial_{x}|f(x)|\right) /|f(x)|\right)^{n} d m(x) \leqq 2^{n+1}(n+1) K^{n-1} \log \lambda m_{n-1}(G) .
$$

Hence $\left(\left(L_{f}-\partial_{x}|f|\right) /|f|\right)^{n}$ is integrable over $C$.

We consider again the extension $\hat{f}$ of $f$ and define a sequence $g_{j}: R^{n} \rightarrow R^{n}$ of quasiconformal mappings by

$$
g_{j}(x)=\hat{f}(j x) / j^{K}, j=1,2, \ldots .
$$

For every $j$ is $K_{o}\left(g_{j} \mid C\right)=K_{O}(f)=K^{n-1}$ and $g_{j}|\partial C=f| \partial C$. By Väisälä [3], 20.5, 21.3, 37.2 and by the above Theorem 2 the sequence $g_{j}$ has a subsequence $g_{j}, j \in J \subset N$, which converges uniformly on compact subsets of $R^{n}$ and whose limit mapping is quasiconformal with $K_{O}(g \mid C)=K^{n-1}$. The formula (3) is still valid for $g$ with the same bound $\lambda$.

$\mathrm{L}$ e $\mathrm{m} \mathrm{m}$ a 3 . The mapping $g$ maps each ray $r_{y}$ onto a ray $r_{z}$.

Proof. Suppose $a<b$. We choose a set $E \subset G, m_{n-1}(E)=m_{n-1}(G)$, such that $g_{j}$ is for every $j \in J$ locally absolutely continuous on every ray $r_{y}, y \in E$. Denote $L_{j}=L_{g_{j}}$. By Fatou's Lemma

$$
\begin{aligned}
& \int_{G} \liminf _{j \rightarrow \infty, j \in J}\left(\int_{a}^{b}\left(L_{j}(t y)-\partial_{y}\left|g_{j}(t y)\right|\right) d t\right)^{n} d m_{n-1}(y) \\
\leqq & \liminf _{j \rightarrow \infty, j \in J} \int_{G}\left(\int_{a}^{b}\left(L_{j}(t y)-\partial_{y}\left|g_{j}(t y)\right|\right) d t\right)^{n} d m_{n-1}(y) .
\end{aligned}
$$

Since $L_{j}(t y)=j^{1-K} L_{f}(j t y)$ and $\partial_{y}\left|g_{j}(t y)\right|=j^{1-K} \partial_{y}|f(j t y)|$, the latter integral is at most

$$
\begin{aligned}
& \int_{G}\left(\int_{a}^{b}\left(L_{j}(t y)-\partial_{y}\left|g_{j}(t y)\right|\right)^{n} t^{n-1} d t\right) d m_{n-1}(y)\left(\int_{a}^{b} t^{-1} d t\right)^{n-1} \\
& =(\log b / a)^{n-1} \int_{C(a, b)}\left(\left(L_{f}(j x)-\partial_{x}|f(j x)|\right) j^{1-K}\right)^{n} d m(x),
\end{aligned}
$$

where $C(a, b)=\{x \in C|a \leqq| x \mid \leqq b\}$. Setting $j x=z \quad$ we obtain

$$
\begin{gathered}
(\log (b / a))^{n-1} \int_{C(j a, j b)}\left(\left(L_{f}(z)-\partial_{y}|f(z)|\right) j^{-K}\right)^{n} d m(z) \\
=(\log (b / a))^{n-1} b^{K n} \int_{C(j a, j b)}\left(\left(L_{f}(z)-\partial_{y}|f(z)|\right)(j b)^{-K}\right)^{n} d m(z) \\
\leqq \lambda^{n} b^{K n}(\log (b / a))^{n-1} \int_{C(j a, j b)}\left(\left(L_{f}(z)-\partial_{y}|f(z)|\right) /|f(z)|\right)^{n} d m(z)
\end{gathered}
$$

for $j b \geqq M$, because then $|f(z)| \leqq \lambda(j b)^{K}$. By Lemma 2 this converges to zero when $j \rightarrow \infty$. Thus a set $E_{1} \subset E$ can be chosen so that $m_{n-1}\left(E_{1}\right)=m_{n-1}(E)$ and 


$$
\liminf _{j \rightarrow \infty, j \in J} \int_{a}^{b}\left(L_{j}(t y)-\partial_{y}\left|g_{j}(t y)\right|\right) d t=0
$$

for every $y \in E_{1}$.

Fix $y \in E_{1}$. For a subsequence $\left(j_{k}\right)$ of $J$

$$
\lim _{k \rightarrow \infty} \int_{a}^{b}\left(L_{j_{k}}(t y)-\partial_{y}\left|g_{j_{k}}(t y)\right|\right) d t=0 .
$$

Now

$$
\int_{a}^{b} \partial_{y}\left|g_{j_{k}}(t y)\right| d t=\left|g_{j_{k}}(b y)\right|-\left|g_{j_{k}}(a y)\right|
$$

converges to $|g(b y)|-|g(a y)|$. Hence the length of the $g_{j_{k}}$-image of the interval $\{t y \mid a \leqq t \leqq b\}$ also converges by (4) to the same limit. Since $g_{j_{k}} \rightarrow g$, an elementary argument implies that the $g$-image of $\{t y \mid a \leqq$ $t \leqq b\}$ is a radial interval. By the continuity of $g$ this holds for every $y \in G$. Since $a$ and $b$ were arbitrary, the lemma follows.

Let $B \subset G$ be a Borel set. By Lemma 3 the $g$-image of the cone $C(B)=\{t y \mid y \in B, t>0\}$ is again a cone. Let $\sigma_{g}(y)$ be the volume derivative of the homeomorphism $h_{g}=P_{r} \circ(g \mid G): G \rightarrow G$, where $P_{r}$ denotes the radial projection of $C$ onto $G$. The number $\sigma_{g}(y)$ is finite for almost every $y \in G$.

$\mathrm{L}$ e $\mathrm{m} \mathrm{m}$ a 4 . The number $\sigma_{g}(y)=1$ a.e. in $G$.

Proof. Let $y \in G$ such that $\sigma_{g}(y)<\infty$. For $\varepsilon>0$ choose $r>0$ so small that $U=B^{n}(y, r) \cap S^{n-1} \subset G \quad$ and

$$
m_{n-1}\left(h_{g}(U)\right)<\left(\sigma_{g}(y)+\varepsilon\right) m_{n-1}(U) .
$$

For arbitrary $t_{1} \leqq 1 / M, \quad t_{2} \geqq M, \quad \lambda t_{1}<t_{2} / \lambda$ we consider the curve family

$$
\Gamma=\Delta\left(t_{1} U, t_{2} U ; C\left(U ; t_{1}, t_{2}\right)\right),
$$

where $C\left(U ; t_{1}, t_{2}\right)=\left\{t z \mid z \in U, t_{1}<t<t_{2}\right\}$. Then

$$
M(T)=m_{n-1}(U) /\left(\log \left(t_{2} / t_{1}\right)\right)^{n-1}
$$

and

$$
M(g \Gamma)=\left(\sigma_{g}(y)+\varepsilon\right) m_{n-1}(U) /\left(\log \left(t_{2}^{K} / \lambda^{2} t_{1}^{K}\right)\right)^{n-1} .
$$

Letting $t_{2} \rightarrow \infty, M(I) \leqq K^{n-1} M(g I)$ now implies

$$
\sigma_{g}(y)+\varepsilon \geqq 1,
$$

and thus, since $\varepsilon>0$ is arbitrary,

$$
\sigma_{g}(y) \geqq 1 .
$$


On the other hand, by the inequality

$$
\int_{G} \sigma_{g}(y) d m_{n-1}(y) \leqq m_{n-1}(G),
$$

$\sigma_{g}(y) \leqq 1$ a.e. in $G$. Thus $\sigma_{g}(y)=1$ a.e. in $G$.

Remark. It will be later proved that also $f$ maps the rays $r_{y}$ onto rays. From the above proof it follows that then also $\sigma_{f}(y)=1$ a.e. in $G$.

$\mathrm{L}$ e $\mathrm{m} \mathrm{ma}$. For almost every $x \in C$

$$
L_{g}(x)=K|g(x)| /|x| .
$$

Proof. Let $B \subset G$ be a Borel set. Denote $C(B ; a, b)=\{t y \mid y \in B$, $a<t<b\}$. Since $h_{g}$ satisfies the condition $(\mathrm{N})$ and $g$ maps the rays onto rays, we have

$$
\begin{gathered}
\int_{C(B ; a, b)} J(x, g) d m(x)=m(g(C(B ; a, b))) \\
=\int_{h_{g} B} d m_{n-1}(v) \int_{|g(a y)|}^{|g(b y)|} u^{n-1} d u \\
=\int_{B} \sigma_{g}(y) d m_{n-1}(y) \int_{a}^{b} t^{n-1}(|g(t y)| / t)^{n-1} \partial_{x}|g(t y)| d t \\
=\int_{C(B ; a, b)}(|g(x)| /|x|)^{n-1} \partial_{x}|g(x)| d m(x) .
\end{gathered}
$$

Thus $J(x, g)=(|g(x)| /|x|)^{n-1} \partial_{x}|g(x)|$ and consequently $L_{g}(x)^{n} \leqq$ $K^{n-1} J(x, g)=K^{n-1}(|g(x)| /|x|)^{n-1} \partial_{x}|g(x)| \leqq K^{n-1}(|g(x)| /|x|)^{n-1} L_{g}(x)$ a.e. in $C$. The lemma follows.

We repeat now the above process with respect to the mapping $g$. We get a sequence $\varphi_{j}: R^{n} \rightarrow R^{n}, j \in J$,

$$
\varphi_{j}(x)=g(j x) j^{-K} .
$$

This sequence has a subsequence $\varphi_{j}, j \in J_{1} \subset J$, which converges uniformly on compact subsets of $R^{n}$, and its limit mapping $\varphi$ is quasiconformal with $K_{O}(\varphi \mid C)=K^{n-1}$. Further $\varphi$ has the boundary values (2) on $\partial C$ and Theorem 1 as well as Lemmas $3-5$ are valid for it. We will prove now that $\varphi$ sends the domain $t G$ onto $t^{K} G$.

$\mathrm{L}$ e $\mathrm{m} \mathrm{m}$ a 6 . For every $x \in C$

$$
|\varphi(x)|=|x|^{K} .
$$

Proof. For a.e. $y \in G$ the mapping $g$ is locally absolutely continuous on the ray $r_{y}=\{t y \mid y \in G, t>0\}$ and $L_{g}(t y) \leqq K|g(t y)| / t$ for almost all $t>0$, see Lemma 5. Choose such $y$. Let $M$ be as in Theorem 1 . Then for $u>a>M$ 


$$
\begin{gathered}
K(\log u-\log a)-2 \log \lambda \leqq \log |g(u y)|-\log |g(a y)| \\
=\int_{a}^{b}\left(\partial_{y}|g(t y)| /|g(t y)|\right) d t
\end{gathered}
$$

and consequently

$$
\lim _{a \rightarrow \infty} \int_{a}^{\infty}\left(K\left|t-\partial_{y}\right| g(t y)|/| g(t y) \mid\right) d t=0,
$$

because the integrand is by Lemma 5 nonnegative; the nonnegativity of the integrand is not yet known for $f$ and this is the main reason for the above process.

For arbitrary $z>0$

$$
\begin{gathered}
K \log z-\log \left(\left|\varphi_{j}(z y)\right| /\left|\varphi_{j}(y)\right|\right)=\int_{1}^{z}\left(K / t-\partial_{y}\left|\varphi_{j}(t y)\right| /\left|\varphi_{j}(t y)\right|\right) d t \\
=\int_{1}^{x}\left(K / t-j \partial_{y}|g(j t y)| /|g(j t y)|\right) d t .
\end{gathered}
$$

Substitution $j t=u$ and (5) yield

$$
\begin{aligned}
& \int_{1}^{z}\left(K / t-j \partial_{y}|g(j t y)| /|g(j t y)|\right) d t \\
&= \int_{j}^{j z}\left(K / u-\partial_{y}|g(u y)| /|g(u y)|\right) d u \\
&=K \log z-\log (|g(j z y)| /|g(j y)|) \rightarrow 0 \text { when } j \rightarrow \infty, \quad j \in J_{1} .
\end{aligned}
$$

Since $\varphi_{j} \rightarrow \varphi$ and $\varphi$ is continuous, the last two formulas give

$$
|\varphi(z y)|=z^{K}|\varphi(y)|
$$

for all $y \in G, z>0$, and consequently

$$
\partial_{y}|\varphi(z y)|=K z^{K-1}|\varphi(y)| .
$$

Since Lemma 5 can also be applied to $\varphi$,

$$
L_{\varphi}(z y) \leqq K|\varphi(z y)| / z=K z^{K-1}|\varphi(y)|
$$

and thus

$$
L_{\varphi}(z y)=\partial_{y}|\varphi(z y)|
$$

for a.e. $z y \in C$. At these points $\operatorname{grad}|\varphi(z y)|=\partial_{y}|\varphi(z y)| y$ and hence $\partial_{s}|\varphi(z y)|=\operatorname{grad}|\varphi(z y)| \cdot s=0$ in every to $y$ orthogonal direction $s$.

This implies that $|\varphi(x)|=|x|^{K}$ in $C$, for if $\left|\varphi\left(x_{1}\right)\right| \neq\left|\varphi\left(x_{2}\right)\right|$ at the points $x_{1}, x_{2} \in C,\left|x_{1}\right|=\left|x_{2}\right|$, then by a theorem of Fuglede (Väi- 
sälä [3], p. 95) the points $x_{1}^{\prime} \in C$ and $x_{2}^{\prime} \in C$ can be chosen such that a) $\left|x_{1}^{\prime}\right|=\left|x_{2}^{\prime}\right|$, b) $\left|\varphi\left(x_{1}^{\prime}\right)\right| \neq\left|\varphi\left(x_{2}^{\prime}\right)\right|$, c) $\varphi$ is absolutely continuous on a regular curve $c$ which lies on the set $\left\{x|x|\left|x_{1}^{\prime}\right| \in G\right\}$ and which joins the points $x_{1}^{\prime}$ and $\left.x_{2}^{\prime}, \mathrm{d}\right)$ the tangential derivative $\partial_{s}|\varphi(x)|$ vanishes a.e. in $C$. The integration leads then to contradiction. Hence $\left|\varphi\left(x_{1}\right)\right|$ $=\left|\varphi\left(x_{2}\right)\right|$ and the boundary condition (2) gives the result.

Remark. The last part of the above proof implies: If $\partial_{s}|f(x)|=0$ a.e. in $C$, where $s$ is any direction orthogonal to $x$, then $|f(x)|=|x|^{K}$ in $C$. This fact will be used later.

$\mathrm{L}$ e $\mathrm{m} \mathrm{m}$ a 7 . There is a sequence $J_{2} \subset N$ such that for every $y \in G$

$$
\lim _{\substack{j \rightarrow \infty \\ j \in J_{2}}}\left|g_{j}(y)\right|=1 \text {. }
$$

Proof. Let $k \in N$. Since $\left|\varphi_{j}(y)\right| \rightarrow|\varphi(y)|=1, \quad j \in J_{1}$, uniformly in $S^{n-1}$, we can choose $i_{k} \in J_{1}$ such that

$$
1-1 / k<\left|\varphi_{i_{k}}(y)\right|<1+1 / k
$$

for every $y \in G$. Further $g_{j} \rightarrow g, j \in J_{1}$, uniformly in $i_{k} S^{n-1}$. Thus there is $j_{k} \in J_{1}$ so that

$$
1-1 / k<\left|g_{j_{k}}\left(i_{k} y\right)\right| /\left|g\left(i_{k} y\right)\right|<1+1 / k
$$

for every $y \in G$. Denote $J_{2}=\left(i_{k} j_{k}\right), k=1,2, \ldots$. Now the proposition follows immediately from

$$
\begin{gathered}
\left|f\left(i_{k} j_{k} y\right)\right|\left(i_{k} j_{k}\right)^{-K}=\left|g_{j_{k}}\left(i_{k} y\right)\right| i_{k}^{-K}= \\
\left|g_{j_{k}}\left(i_{k} y\right)\right|\left|g\left(i_{k} y\right)\right|^{-1}\left|g\left(i_{k} y\right)\right| i_{k}^{-K}= \\
\left|g_{j_{k}}\left(i_{k} y\right)\right|\left|g\left(i_{k} y\right)\right|^{-1}\left|\varphi_{i_{k}}(y)\right| .
\end{gathered}
$$

The mapping $h=I \circ \hat{f} \circ I: R^{n} \rightarrow R^{n}$, where $I$ is the inversion $I(x)=x /|x|^{2}$, satisfies the conditions $K_{O}(h \mid C)=K_{O}(f)$ and $h \mid \partial C=$ $f \mid \partial C$. The above results can thus also be applied to $h$ and to respective sequences. If we denote

$$
g_{1 / j}(y)=j^{K} f(y / j)
$$

then we see immediately the validity of the following lemma.

$\mathrm{L}$ e $\mathrm{m} \mathrm{ma}$. There is a subsequence $J_{3}$ of $J_{2}$ such that for every $y \in G$

$$
\lim _{\substack{j \rightarrow \infty \\ j \in J_{3}}}\left|g_{j}(y)\right|=1, \lim _{\substack{j \rightarrow \infty \\ j \in J_{3}}}\left|g_{1 / j}(y)\right|=1 .
$$

Now we are ready to prove Theorem 3 .

Proof of Theorem 3. We show first that 


$$
\int_{C}\left(L_{f}(x)^{n}-\left|\partial_{x}\right| f(x)||^{n}\right)|f(x)|^{-n} d m(x)=0 .
$$

Denote for $j \in J_{3}$

$$
\begin{aligned}
& a_{j}(y)=\min _{x \in G}\left\{\left|g_{1 / j}(x)\right| \mid P_{r} f(x / j)=y\right\}, \\
& b_{j}(y)=\max _{x \in G}\left\{\left|g_{j}(x)\right| \mid P_{r} f(j x)=y\right\},
\end{aligned}
$$

where $P_{r}$ is the radial projection of $R^{n}$ onto $S^{n-1}$. Since

$$
|f(j y)|=\left|g_{j}(y)\right| j^{K},|f(y / j)|=\left|g_{1 / j}(y)\right| / j^{K},
$$

we obtain as in the proof of Lemma 1

$$
\begin{gathered}
\int_{G}\left(2 K \log j+\log \left|g_{j}(y)\right|-\log \left|g_{1 / j}(y)\right|\right)^{n} d m(y) \\
\leqq(2 \log j)^{n-1} \int_{C_{j}}\left|\partial_{x}\right| f(x)||{ }^{n}|f(x)|^{-n} d m(x) \\
\leqq(2 \log j)^{n-1} \int_{C_{j}} L_{f}(x)^{n}|f(x)|^{-n} d m(x) \\
\leqq(2 K \log j)^{n-1} \int_{G} d m_{n-1}(y) \int_{a_{j}(y) / j^{K}}^{b_{j}(y) j^{K}} t^{-1} d t \\
=(2 K \log j)^{n-1} \int_{G}\left(2 K \log j+\log b_{j}(y)-\log a_{j}(y)\right) d m_{n-1}(y) .
\end{gathered}
$$

Hence

$$
\begin{aligned}
& 0 \leqq \int_{C_{j}}\left(L_{f}(x)^{n}-\left|\partial_{x}\right| f(x)||^{n}\right)|f(x)|^{-n} d m(x) \\
& \leqq K^{n-1} \int_{G}\left(\log b_{j}(y)-\log a_{j}(y)\right) d m_{n-1}(y) \\
&-\sum_{k=1}^{n}\left(\begin{array}{l}
n \\
k
\end{array}\right) K^{k}(2 \log j)^{k-n+1} \int_{G}\left(\log \left|g_{j}(y)\right|-\log \left|g_{1 / j}(y)\right|\right)^{n-k} d m_{n-1}(y) .
\end{aligned}
$$

Since $g_{j} \rightarrow 1, \quad g_{1 / j} \rightarrow 1, j \in J_{3}$, uniformly on $S^{n-1}$, we have for every $y \in G$

$$
\lim _{\substack{j \rightarrow \infty \\ j \in J_{3}}} a_{j}(y)=\lim _{\substack{j \rightarrow \infty \\ j \in J_{3}}} b_{j}(y)=1 .
$$

Moreover $\left|g_{j}(y)\right| \leqq \lambda,\left|g_{1 / j}(y)\right| \geqq 1 / \lambda, b_{j}(y) \leqq \lambda, a_{j}(y) \geqq 1 / \lambda$ for $j \geqq \mathrm{M}$, hence by the Lebesgue convergence Theorem

$$
\int_{C}\left(L_{f}(x)^{n}-\left|\partial_{x}\right| f(x)||^{n}\right)|f(x)|^{-n} d m(x)=0 .
$$

Thus $L_{f}(x)=\left|\partial_{x}\right| f(x)||$ and consequently $\partial_{s}|f(x)|=0$ a.e. in $C$ for every direction $s$ orthogonal to $x$. Remark after Lemma 6 implies that 
$f$ maps every domain $G(t)$ onto $G\left(t^{K}\right)$. Thus $L_{f}(x)=\partial_{x}|f(x)|=K|x|^{K-1}$ for a.e. $x \in C$, and the length of the image of an arbitrary segment $\{t y \mid a \leqq t \leqq b\}$ is for a.e. $y \in G$ equal to $b^{K}-a^{K}$, i.e. the distance between $G\left(a^{K}\right)$ and $G\left(b^{K}\right)$. Now $P_{r}(f(a y))=P_{r}(f(b y))$ and by the continuity of $f$ even for all $y \in G$. This means that $f$ carries every ray $r_{y}$ onto a ray. Finally, by Remark after Lemma $4 \sigma_{f}(x)=1$ a.e. in $G$. The theorem is proved.

We show now that in the case $K_{0}(f)=K^{n-1}$ the mapping $f$ need not be defined by (2) in $C$.

Example. Let $w(z)=e^{i} z e^{-2 i|z|}$ be a quasiconformal mapping of the plane disc $D=\{z|| z \mid<1 / 2\}$ onto itself. Then $w(z)=z$ on the boundary of $D$ and $J(z, w)=1$ for every $z \in D$, see $[2$, p. 17]. Let

$$
G=\left\{x \in R^{3} \mid x_{1}^{2}+x_{2}^{2}+x_{3}^{2}=1, x_{1}^{2}+x_{2}^{2}<1 / 4, x_{3}>0\right\}
$$

and let $P$ be the orthogonal projection of $G$ onto $D$. Then the composed mapping $\varphi=P^{-1} \circ w \circ P: G \rightarrow G$ also satisfies the conditions $\varphi(x)=x$ for $x \in \partial G$ and the area derivative $\sigma(x, \varphi)=1$ for $x \in G$, because $|w(z)|=|z|$ for every $z \in D$. Denote the supremum of the maximal stretch$\operatorname{ing} L_{\varphi}(y)$ by $M$, where the supremum is taken over all $y \in G$.

We define now the mapping $f: C \rightarrow C$ by

$$
f(x)=\varphi(x /|x|)|x|^{K},
$$

where $K>M$ is a constant. Then $f(x)=|x|^{K-1} x$ on the boundary of $C$ and the volume derivative at $x$ is

$$
K|x|^{K-1}\left(|x|^{K-1}\right)^{2} \sigma(x /|x|, \varphi)=K|x|^{3(K-1)} .
$$

Since $K>M$, the maximal stretching of $f$ at $x$ is $K|x|^{K-1}$. Thus

$$
K_{0}(f)=K^{2},
$$

but the mapping $\varphi$ is not the identity mapping.

Th e or em 4. If $K_{I}(f)=K$, then $f$ is composed of the mapping (2) and a rotation.

Proof. Theorem 2 and the inequality $K_{O}(f) \leqq K_{I}(f)^{n-1}$ imply $K_{O}(f)$ $=K^{n-1}$ and hence Theorem 3 is valid. Let $x \in C$ be a regular point of $f$ such that $\sigma_{f}\left(P_{r}(x)\right)=1$. Let $\lambda_{1}=\left|\partial_{x} f(x)\right|=K|x|^{K-1}, \lambda_{2}, \ldots, \lambda_{n}$ be the semiaxis of the dilatation ellipsoid $E\left(f^{\prime}(x)\right), \quad \lambda_{2} \geqq \ldots \geqq \lambda_{n}$. By Theorem 3

$$
\lambda_{2} \ldots \lambda_{n}=|x|^{(n-1)(K-1)}
$$

and hence $\lambda_{n} \leqq|x|^{K-1}$. The definition of $K_{I}(f)$ gives

$$
\lambda_{1} \ldots \lambda_{n}=K|x|^{K-1}|x|^{(n-1)(K-1)} \leqq K \lambda_{n}^{n},
$$


i.e. $\lambda_{n} \geqq|x|^{K-1}$. Thus $\lambda_{n}=|x|^{K-1}$. The equality (6) implies then, since $\lambda_{2} \geqq \ldots \geqq \lambda_{n}$,

$$
\lambda_{2}=\lambda_{3}=\ldots=\lambda_{n}=|x|^{K-1} .
$$

Hence $f \mid G$ is a rotation, and the theorem follows.

Remark. The rotation in Theorem 4 can appear only if $\partial C$ is contained in an (n-2)-dimensional linear subspace of $R^{n}$.

\section{References}

[1] Rickman, S.: Removability theorems for quasiconformal mappings. - Ann. Acad. Sci. Fenn. Ser. A I 449, 1969, 1-8.

[2] TAARI, O.: Einige Extremalprobleme für n-dimensionale quasikonforme Abbildungen. - Ann. Acad. Sci. Fenn. Ser. A I 553, 1973, 1-18.

[3] VÄIs̈̈цё, J.: Lectures on $n$-dimensional quasiconformal mappings. - Lecture Notes in Mathematics 229, Springer-Verlag, Berlin - Heidelberg New York, 1971.

[4] V̈̈ıs̈̈цӓ, J.: Piecewise quasiconformal maps are quasiconformal. - Ann. Acad. Sci. Fenn. Ser. A I 1, 1975, 3-6.

University of Helsinki

Department of Mathematies

SF-00100 Helsinki 10

Finland

Received 6 November 1974 